\title{
Involvement of sperm acetylated histones and the nuclear isoform of Glutathione peroxidase 4 in fertilization
}

\author{
Simona Pipolo ${ }^{1}$ | Rossella Puglisi ${ }^{1}$ | Valentina Mularoni ${ }^{1}$ | Valentina Esposito ${ }^{1}$ \\ Andrea Fuso $^{2}$ | Marco Lucarelli ${ }^{3}$ | Maria T. Fiorenza ${ }^{4}$ | Franco Mangia ${ }^{4}$ | \\ Carla Boitani ${ }^{1}$
}

1 Department of Anatomical, Histological, Forensic and Orthopedic Sciences - Section of Histology and Medical Embryology, Sapienza University of Rome, Rome, Italy

2 Department of Surgery "P. Valdoni”, Sapienza university of Rome, Rome, Italy

${ }^{3}$ Department of Cellular Biotechnologies and Hematology and Pasteur Institute, CenciBolognetti Foundation, Sapienza University of Rome, Rome, Italy

${ }^{4}$ Department of Psychology, Sapienza University of Rome, Rome, Italy

\section{Correspondence}

Carla Boitani, Associate Professor of Histology, DAHFMO- Section of Histology and Medical Embryology, University of Rome "La Sapienza", via A. Scarpa 14, 00161 Roma, Italy.

Email: carla.boitani@uniroma1.it

Funding information

Italian Ministry of Education

\begin{abstract}
We previously demonstrated that the nuclear form of Glutathione peroxidase 4 ( $n G P x 4$ ) has a peculiar distribution in sperm head, being localized to nuclear matrix and acrosome and that sperm lacking nGPx4 are more prone to decondensation in vitro. In this study we have hypothesized that sperm retained acetylated histones and nGPx4 are implicated in paternal chromatin decondensation and male pronucleus formation at fertilization. Indeed, significant higher amounts of acetylated histone $\mathrm{H} 4$ and acetylated histone $\mathrm{H} 3$ were observed by both immunofluorescence and western blotting in nGPx4-KO sperm vs WT ones. In vitro fertilization of zona pellucidadeprived oocytes by WT sperm in the presence of trichostatin (TSA) also demonstrated that paternal histone acetylation was inversely related to the timing of sperm nucleus decondensation at fertilization. In contrast, TSA had no effect on nGPx4-KO sperm, indicating they had a maximal level of histone acetylation. Moreover the paternally imprinted gene Igf $2 / \mathrm{H} 19$ was hypomethylated in KO sperm compared to WT ones. The lack of nGPx4 negatively affected male fertility, causing a marked decrease in total pups and pregnancies with delivery, a significant reduction in pronuclei (PN) embryos in in vitro fertilization assays and an approximately $2 \mathrm{~h}$ delay in egg fertilization in vivo. Because the zona pellucida binding and fusion to oolemma of nGPx4-KO and WT sperm were similar, the subfertility of nGPx4 sperm reflected a decreased sperm progression through egg cumulus/zona pellucida, pinpointing a defective acrosome in line with acrosomal nGPx4 localization. We conclude that paternal acetylated histones and acrosomal nGPx4 are directly involved in fertilization.
\end{abstract}

KEYWORDS

male fertility, $\mathrm{nGPx} 4$, sperm chromatin remodeling, sperm retained histones
Present address of Simona Pipolo: Istituto clinico Humanitas, Rozzano, Milan, Italy. Present address of Rossella Puglisi: Centro di Medicina di Genere, Istituto Superiore di Sanità, Rome, Italy.

\section{1 | INTRODUCTION}

The mature mammalian sperm chromatin has a unique structure and composition. Most of sperm DNA is coiled into tightly compacted toroids by protamines, highly basic sperm specific proteins, and forms loop domains attached to the nuclear matrix at regularly 
spaced matrix attachment regions (MARs) (Puglisi et al., 2012; Ward, 2010). The protamine-DNA assembly is accomplished during spermiogenesis through a progressive histone-to-protamine replacement that leads to extensive nuclear remodeling and consists in the prior destabilization of nucleosomes followed by the exchange of histones first with transition proteins and then with protamines (Puglisi et al., 2012). During spermiogenesis, this process is accompanied by extensive histone acetylation, as indicated by the presence of hyperacetylated histone $\mathrm{H} 4$ in mouse and human elongating spermatids from steps 9 to 11 , that seemingly disappear at later sperm maturationxxxx stages (Hazzouri et al., 2000; Sonnack, Failing, Bergmann, \& Steger, 2002). However, the process of histone substitution with protamine is not complete, because a small percentage of histones bound to DNA in the nucleosomal configuration (between $1 \%$ and $15 \%$, depending on the species) (Wykes \& Krawetz, 2003) is actually retained in the mature sperm. In fact using unmasking procedures followed by immunofluorescence, the presence of acetylated histone $\mathrm{H} 4$ and of acetylated histone $\mathrm{H} 3$ was revealed in decondensed sperm nuclei from mouse caput epididymis (van der Heijden et al., 2006) and human ejaculated spermatozoa (Steilmann et al., 2011), respectively. More recently, the presence of histones in mature human and mouse sperm has been confirmed by mass spectrometry and western blot analyses, also identifying several post-translational histone modifications, including acetylation (Brunner, Nanni, \& Mansuy, 2014; Krejci et al., 2015; Luense et al., 2016).

It comes as no surprise the massive attention the retention of histones in the mature male gamete has recently received. The sperm retained histones and their modifications, including acetylation, methylation, phosphorylation, and ubiquitination, have been recognized as epigenetic marks that regulate the expression of a subset of genes involved in the early embryonic development (Arpanahi et al., 2009; Brykczynska et al., 2010; Hammoud et al., 2009; Jenkins \& Carrell, 2012). Besides histones, the sperm epigenome also includes DNA methylation, non-coding RNA and RNA modifications (Chen, Yan, \& Duan, 2016). Of great interest, recent studies in the mouse reported evidence of paternal epigenetic transmission across generations mediated by histone $\mathrm{H} 3$ methylation (Siklenka et al., 2015) or non coding RNAs (Rodgers, Morgan, Leu, \& Bale, 2015), pointing to the potential impact that a defective sperm epigenome, caused by environmental/lifestyle factors or aging, may have on the phenotype and disease susceptibility not only of the offspring but also the following generations. Among several pathologies, male infertility is associated with epigenetic errors (Rajender, Avery, \& Agarwal, 2011). Indeed, an aberrant DNA methylation pattern was observed in sperm from infertile patients displaying abnormal protamination or oligozoospermia/oligo-astheno-teratozoospermia (Boissonnas et al., 2010; Hammoud, Purwar, Pflueger, Cairns, \& Carrell, 2010).

After male gamete penetration into oocyte at fertilization, sperm chromatin undergoes a striking remodeling initiated by decondensation, which finally leads to male pronucleus formation. In this process sperm protamines are rapidly released from DNA and substituted by the histones of the egg, resulting in de novo assembly of regular nucleosomes. How this process occurs is only partially known. A relevant role in the deposition of maternal histones onto paternal DNA is played by histone chaperone HIRA, as demonstrated in Drosophila and mouse sperm nucleus decondensation after gamete fusion (Bonnefoy, Orsi, Couble, \& Loppin, 2007; van der Heijden et al., 2005). In addition, it was also established that the histones of paternal origin are released into the oocyte (van der Heijden et al., 2006), although the male origin was initially misinterpreted in immunostaining studies on the zygote due to the concomitant deposition of maternally derived histones (Adenot, Mercier, Renard, \& Thompson, 1997; Santos, Hendrich, Reik, \& Dean, 2002; Santos, Peters, Otte, Reik, \& Dean, 2005; van der Heijden et al., 2005). However, whether the paternally derived histones play a functional role in modulating sperm chromatin structural changes after gamete fusion is not yet established. In the present study we have addressed this question by exploiting male gametes of mice knockout (KO) for the nuclear isoform of the selenoprotein Phospholipid Hydroperoxide Glutathione Peroxidase (nGPx4), an enzyme containing several cysteines and able to oxidize protein thiols, besides glutathione, by cross-linking protamines and itself in sperm (Conrad et al., 2005; Puglisi et al., 2012). Being mostly localized to the nuclear matrix of male haploid germ cells and cauda epididymal sperm, nGPx4 is required for the structural integrity of sperm chromatin (Puglisi et al., 2012). Using nGPx4-KO mice, we have been able to correlate the abundance of acetylated sperm histones with chromatin decondensation, pinpointing the functional importance of acetylated histones of paternal origin as early mediators of male chromatin decondensation in the zygote. We also report that $\mathrm{nGPx} 4-\mathrm{KO}$ sperm have an impaired fertilizing ability, revealing a novel contribution of the nuclear GPx4 isoform to male fertility, besides the essential role for sperm motility played by the mitochondrial GPx4 variant (Schneider et al., 2009).

\section{2 | MATERIALS AND METHODS}

\section{$2.1 \mid$ Animals and genotyping}

C57BL/6J, CD1 mice (Charles River Laboratories, Italy) and KO C57BL/6J mice specifically lacking nGPx4 (kindly provided by Dr.Marcus Conrad, Institute of Developmental Genetics, Neuherberg, Germany) were used in this study. The $\mathrm{nGPx} 4$ colony was maintained by crossing $\mathrm{nGPx} 4^{+/-}$and $\mathrm{nGPx} 4^{-/-}$mice. Genotyping was performed by polymerase chain reaction (PCR) of genomic DNA extracted from tail biopsies, using the following primer pairs (forward/reverse): I1 $\mathrm{f} 2 /$ Earev1, (5'-TCGGCGGCGCCTTGGCTACCGGCTC-3'/5'-GGATCCGC CGCGCTGTCTGCAGCGTCCC-3'), specific for the wild type allele; and I1f2/eGFPrev, (5'-TGAAGAAGTCGTGCTGCT TCATGTGG-3'), specific for the KO allele, as previously described (Conrad et al., 2005). Animals were housed in the Histology Department accredited animal facility. All experimental procedures were approved by the Italian Ministry of Health and were conducted according to Sapienza University guidelines. 


\section{2 | Immunofluorescence and sperm nucleus decondenzation analysis}

Cauda epididymal spermatozoa were collected from adult wild-type (WT) and homozygous nGPx4-KO mice and capacitated at $37^{\circ} \mathrm{C}$ for $90 \mathrm{~min}$, as previously described (Luconi et al., 2005). Sperm nucleus decondensation was performed as previously described (Puglisi et al., 2012). Briefly, sperm were incubated in the presence of $5 \mathrm{mM}$ glutathione (GSH) and $10 \mu \mathrm{M}$ heparin (Sigma-Aldrich, St Louis, MO) in $\mathrm{T} 6$ medium supplemented with $1 \%$ Triton $\mathrm{X}-100$ at $37^{\circ} \mathrm{C}$ for increasing times. At each time period a sperm aliquot was removed, fixed with $4 \%$ paraformaldehyde (PFA) in PBS for $10 \mathrm{~min}$ at $4^{\circ} \mathrm{C}$ and cytocentrifuged on polysine coated slides. For immunostaining, cells were incubated for $30 \mathrm{~min}$ in PBS containing 5\% BSA and $0.1 \%$ Triton $\mathrm{X}-100$. After washing cells were treated overnight at $4^{\circ} \mathrm{C}$ with the following primary antibodies: rabbit anti-acetyl-histone $\mathrm{H} 4$ (1:100, Merck-Millipore, Darmstadt, Germany), rabbit anti-acetylhistone H3 (1:100, Merck-Millipore) and goat anti-protamine-2 (1:100, Santa Cruz Biotechnology, Heidelberg, Germany) in PBS containing 1\% BSA and 0.1\% Triton X-100 (PBT). Cells were then incubated for $1 \mathrm{~h}$ at room temperature with the appropriate secondary antibodies: anti-rabbit Alexa Fluor 488-conjugated antibody (1:400 Molecular Probes, Leiden, The Netherlands), antirabbit Alexa Fluor 555-conjugated antibody (1:500 Molecular Probes) and anti-goat Alexa Fluor 555-conjugated antibody (1:1000, Molecular Probes). TO-PRO-3 (1:1000, Molecular Probes) was used to stain DNA. Slides were mounted with Vectashield (Vector Laboratories, Burlingame, CA) and analyzed under a Zeiss widefield fluorescence microscope. The fluorescence intensity of acetylated histone $\mathrm{H} 4$ and acetylated histone $\mathrm{H} 3$ in "medium" decondensed sperm nuclei (Puglisi et al., 2012) was measured with Image J software (Fiorenza et al., 2008).

\subsection{Protein preparation and Western blot analysis}

Cauda epididymal spermatozoa were incubated in water with $10 \mathrm{mM}$ DTT and protease inhibitors for $30 \mathrm{~min}$ at $37^{\circ} \mathrm{C}$, sonicated at $4^{\circ} \mathrm{C}$ for $5 \mathrm{~min}$ (40\% amplitude, pulse $30 \mathrm{sec}$, pause $15 \mathrm{sec}$ ) and then passed through a 26-gauge needle for six times. Acid soluble proteins were extracted in $0.25 \mathrm{~N}$ hydrochloric acid $(\mathrm{HCl})$ and precipitated with $3.5 \%$ trichloroacetic acid, washed with acidified acetone followed by acetone, then dried and dissolved in water. Protein concentration was determined by the bicinchoninic acid method (Pierce Chemical, Rockford, IL) using albumin as standard. Proteins were fractionated by $18 \%$ acid urea-polyacrylamide gel electrophoresis (AU-PAGE) (Meistrich, 1989) and then transferred onto polyvinylidene fluoride membrane (Immobilon - $\mathrm{P}^{\mathrm{SQ}}$, Merck-Millipore). After saturation, the membranes were incubated overnight at $4^{\circ} \mathrm{C}$ in $5 \%$ nonfat dry milk and $0.1 \%$ Tween-20 with the following primary antibodies: rabbit anti-acetyl histone H4 (1:2000, Merck-Millipore), rabbit anti-acetyl histone H3 (1:1000, Merck- Millipore), mouse monoclonal antiunmodified H3 (1:200, Merck-Millipore), and rabbit anti-mitochondrial GPx4 (1:1000, Abcam, Cambridge, UK). After several washes filters were incubated with the appropriate secondary antibody: antirabbit horseradish peroxidase-conjugated antibody (1:3000, Molecular probes) and anti-mouse horseradish peroxidase-conjugated antibody (1:1000, DAKO, Glostrup, Denmark). Bands were visualized by an enhanced chemiluminescence system (GE Healthcare, Little Chalfont, UK) or diaminobenzidine (DAB) according to manufacturer's recommendations. Mitochondrial GPx4 (mGPx4) was used to normalize histone levels by densitometric analysis with AIDA image analyzer software.

\section{4 | TSA treatment and sperm chromatin decondensation assay}

For chromatin condensation assessment of TSA treated sperm, cumulus-enclosed metaphase II oocytes from hormone-primed CD1 female mice were treated with $0.3 \mathrm{mg} / \mathrm{ml}$ hyaluronidase in $\mathrm{MH}$ to remove cumulus cells and then with Tyrode solution (Nagy, Gertsenstein, Vintersten, \& Behringer, 2003) to remove the zona pellucida (ZP). After washing, oocytes were incubated in the presence or absence of $100 \mathrm{ng} / \mathrm{ml} \mathrm{TSA}$ (Millipore) in HTF medium (Nagy et al., 2003) at $37^{\circ} \mathrm{C}$ for $30 \mathrm{~min}$, preloaded with Hoechst 33342 for $30 \mathrm{~min}$ and then incubated with sperm from WT and nGPx4 KO mice for further $60 \mathrm{~min}$. In these experiments, sperm concentration was adjusted to $10^{4} / \mathrm{mL}$ in the fertilization dish in order not to exceed 3-4 spermatozoa inside the oocyte. After fixation with 4\% PFA, embryos were mounted in toto and fluorescence was analysed under a widefield Zeiss microscope. Spermatozoa were classified according to nucleus refractivity, granular aspect and size in four decondensation classes: "none", "low", "medium", and "high", as previously described (Puglisi et al., 2012). Sperm numbers were expressed for each class of morphology as percentage of total cells examined in five separate experiments.

\section{5 | Analysis of DNA methylation by bisulphite modification and genomic sequencing}

DNA methylation was investigated as previously described (Fuso, Scarpa, Grandoni, Strom, \& Lucarelli, 2006; Fuso et al., 2011) by using MIP primers (Fuso, Ferraguti, Scarpa, Ferrer, \& Lucarelli, 2015). Briefly, bisulphite analysis of Igf2/H19 DMR methylation was performed using the EpiTect Bisulphite kit and PCR products obtained after bisulphite treatment were cloned using the PCR Plus Cloning Kit (both from Qiagen, Hilden, Germany). At least ten clones were analyzed per experimental condition using M13 primers for sequencing. Sequencing reactions were performed on purified plasmid DNA. Clones were sequenced by the cycle sequencing method using the ABI PRISM 3130xl genetic analyzer (Applied Biosystems, Monza, Italy).

Primer names, sequence and position and expected products used for bisulphite analysis are reported in supplementary Table $\mathrm{S} 1$. These primers allowed to assess the methylation status of plus $\left(5^{\prime}->3^{\prime}\right)$ DNA strand on the reference GenBank sequence AY849916.1 (Mus musculus strain $\mathrm{C} 57 \mathrm{BL} 6 / \mathrm{J}$ lgf2/H19 imprinting control region 
sequence). "Fragment $A$ " and "Fragment B" identify two non-adjacent DNA sequences in the Igf2/H19 DMR region; these sequences were chosen to design two different set of primer pairs for the study of DNA methylation. Positive and negative controls were performed as previously described (Fuso et al., 2015).

\section{6 | Fertility assessment of male mice}

WT $(n=6)$ and nGPx4-KO ( $n=6), 12-16$ week-old male mice were mated with CD1 females (one male with two females/cage) for 6 weeks. Females were checked for vaginal plug each morning. Plugpositive females were separated from the male and substituted with new ones until each male had plugged a maximum of six females. Fertility was assessed by recording the number of plugs, the number of productive plugs (plugs followed by pregnancy and delivery) and the size of litters.

\section{7 | In vitro culture of embryos derived from in vivo fertilization}

Four to eight week-old female $\mathrm{CD} 1$ mice were induced to superovulate by intraperitoneal injections of $5 \mathrm{UI} \mathrm{PMSG}$, followed by $5 \mathrm{UI} \mathrm{hCG} 48 \mathrm{~h}$ later, and then mated with WT or nGPx4 males (one male with two females/ cage). At $13 \mathrm{~h}$ after hCG injection, plugged females were sacrificed and embryos were collected from the oviducts and in vitro cultured in M2 medium (Nagy et al., 2003) for $12 \mathrm{~h}$. In vitro embryo development was monitored under stereomicroscope every $30 \mathrm{~min}$, recording the percentage of embryos that had reached the pronuclear stage.

\section{8 | In vitro fertilization with cumulus-enclosed oocytes}

Four to eight week-old female CD1 mice were induced to superovulate by intraperitoneal injections of $5 \mathrm{UI} \mathrm{PMSG}$, followed by $5 \mathrm{UI} \mathrm{hCG} 48 \mathrm{~h}$ later. Females were sacrificed the next day at 9:00 a.m. and the cumulus-oocyte complexes were collected from oviduct ampullae. Sperm were obtained from WT and nGPx4-KO mice of 10-20 weeks. The two cauda epididymides were minced in $1 \mathrm{~mL}$ of HTF medium and were then left for $1.5 \mathrm{~h}$ at $37^{\circ} \mathrm{C}$ to allow sperm swimming-out and capacitation. Hereafter $10 \mu \mathrm{l}$ of sperm suspension was added to oocytes in a fertilization dish. After $4 \mathrm{~h}$ incubation at $37^{\circ} \mathrm{C}$, embryos were washed and cultured for further $4 \mathrm{~h}$ in KSOM under mineral oil for monitoring their development. Fertilization rate was assessed as the percentage of embryos at pronuclear stage.

\section{9 | Sperm binding assay with zona pellucida-enclosed oocytes and sperm fusion assay with zona pellucida-free oocytes}

The in vitro sperm-egg binding assay was performed as previously described (Lin, Roy, Yan, Burns, \& Matzuk, 2007). Briefly, 4-8 week-old $\mathrm{C} 57 \mathrm{BL} / 6 \mathrm{~J}$ female mice were superovulated and sacrificed the next day at 9:00. Metaphase II oocytes were collected from tubes, treated with $0.3 \mathrm{mg} / \mathrm{mL}$ hyaluronidase in $\mathrm{MH}$ to remove cumulus cells and fixed with $2 \%$ PFA in-PBS-PVP (PBS with $0.4 \%$ polyvinylpyrrolidone). Cauda epididymal sperm were obtained as described before, then capacitated at $37^{\circ} \mathrm{C}$ for $1.5 \mathrm{~h}$ and adjusted to a concentration of $10^{5} / \mathrm{mL}$. WT and nGPx4-KO sperm were incubated with cumulus-free oocytes at $37^{\circ} \mathrm{C}$ for $1 \mathrm{~h}$. After fixation with $2 \%$ PFA-PBS-PVP, the number of spermatozoa tightly bound to zona pellucida (ZP) was counted the widest egg diameter under a Wild stereomicroscope.

The in vitro sperm-egg fusion assay was performed as previously described (Puglisi et al., 2012). Oocytes were treated to remove both cumulus cells and zona pellucida. Zona-free oocytes were preloaded with Hoechst 33342 for 30 min, washed three times and incubated with capacitated epididymal sperm (final concentration of $10^{4} / \mathrm{mL}$ ) obtained from WT and nGPx4-KO mice. Embryos were washed $1 \mathrm{~h}$ after insemination, fixed with $2 \%$ PFA in $M 2$ medium and whole mounted for the analysis of oolemma fused sperm, identified by their Hoechst fluorescence staining.

\subsection{0 | Statistical analysis}

All experiments were performed at least in three replicates.

Statistical analyses were performed using the following software: STATISTICA v.8.0 (DNA methylation and sperm chromatin decondensation data); (SigmaPlot v.11.0, Systat, Milan, Italy) and (GraphPad Prism 7, GraphPad Software, La Jolla, CA) (logistic curves analyses); Microsoft Excel 2016 (unpaired Student's $t$-test, all other data). See figure legends for details.

\section{3 | RESULTS}

\subsection{Mouse cauda epididymal sperm physiologically carry a limited amount of acetylated histones $\mathrm{H} 4$ and $\mathrm{H} 3$}

To investigate the role(s) played by modified histones in mouse sperm chromatin, we first determined the presence of acetylated histones by immunofluorescence in cauda epididymal sperm. In these sperm, protamines can be visualized by direct immunostaining, whereas histones are not because they are few and masked by the compacted chromatin. To solve this difficulty, we preliminarily incubated cauda epididymal sperm with glutathione (GSH) and heparin for increasing times in vitro (Puglisi et al., 2012), allowing sperm chromatin to progressively swell depending on incubation duration. This treatment gives rise to a variety of nuclear decondensation features grouped into the arbitrary classes "none," "low," "medium," and "high" according to phase contrast morphology and DNA staining with Hoechst (Puglisi et al., 2012). In this experiment, we incubated sperm of WT mice with GSH and heparin for $45 \mathrm{~min}$ and then performed a double immunostaining with anti-protamine 2 and anti-acetyl-histone $\mathrm{H} 4$ antibodies (Figure 1). Both signals became progressively apparent with increasing nuclear swelling, maximizing in the "medium" class and then decreasing in the "high" class likely because acetylated histones and 


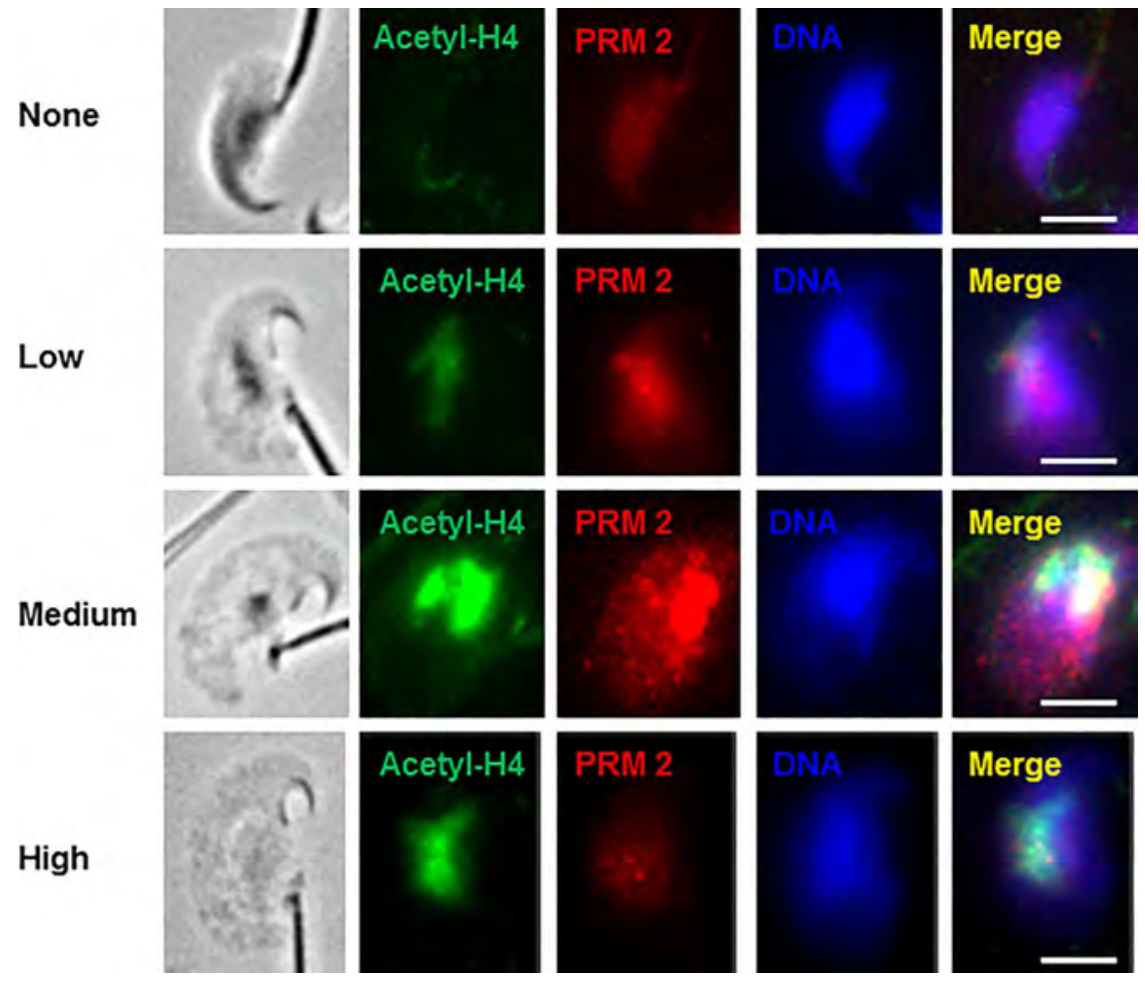

FIGURE 1 The presence of acetylated histone $\mathrm{H} 4$ in cauda epididymal sperm is progressively revealed by in vitro chromatin decondensation. Sperm from WT mice were first incubated with the decondensing reagents glutathione and heparin as described under materials and methods, then either analyzed by phase contrast microscopy (1st column) or by widefield fluorescence microscopy following triple staining with anti-acetyl-H4 antibody ( 2 nd column), anti-protamine 2 antibody (3rd column) and TO-PRO-3 for DNA (4th column). Spermatozoa shown in the figure are representative of the four chromatin decondensation classes (lines): no decondensation (none); low decondensation (low), medium decondensation (medium), and high decondensation (high). Scale bars: $10 \mu \mathrm{m}$

protamines were stripped away by excessive incubation. The "medium" class was therefore selected for further determinations. Similar results were also obtained with anti-acetyl-histone $\mathrm{H} 3$ antibody (data not shown). We therefore concluded that mouse mature sperm prior to fertilization physiologically contain acetylated histones $\mathrm{H} 4$ and $\mathrm{H} 3$, which require nucleus swelling for in situ visualization by immunofluorescence.

\section{2 | Acetylated histones, but not the unmodified ones, are retained at higher extent in sperm with abnormal chromatin condensation}

We next investigated the relationship, if any, between a defective nuclear condensation and the presence of acetylated histones in mature sperm. To this end we analyzed sperm from nGPx4-KO mice, having defective chromatin compaction (Conrad et al., 2005; Puglisi et al., 2012). WT and nGPx4-KO cauda epididymal sperm were treated as described above and then the presence of acetylated histones $\mathrm{H} 3$ and $\mathrm{H} 4$ was revealed by immunofluorescence and quantified in the "medium" decondensed sperm (Figure 2). KO sperm displayed a level of acetylated histones $\mathrm{H} 3$ and $\mathrm{H} 4$ significantly higher than that of WT sperm, indicating that the insufficient compaction of chromatin was accompanied by abnormally high levels of acetylated histones in the mature sperm. This relationship was further investigated by western blot analysis of acetylated histones $\mathrm{H} 3$ and $\mathrm{H} 4$ and unmodified histone $\mathrm{H} 3$ in WT and nGPx4-KO sperm (Figure 3). In agreement with morphological observations, acetylated histones $\mathrm{H} 3$ and $\mathrm{H} 4$ were significantly more abundant in nGPx4-KO than in WT sperm, whereas there was no difference in the unmodified histone $\mathrm{H} 3$ content of the two sperm genotypes. These findings indicate that a specific posttranslational modification of histones as acetylation provides a mark of incomplete sperm nuclear compaction.

\section{3 | An increase in sperm histone acetylation causes a faster chromatin decondensation of WT, but not nGPx4-KO, sperm at fertilization}

The observation that nGPx4-KO cauda epididymal sperm carry abnormally high levels of acetylated histones prompted us to address the question whether they were involved in early sperm chromatin remodeling after fertilization. To this end we experimentally manipulated the level of sperm histone acetylation by the histone deacetylase inhibitor trichostatin (TSA). Cauda epididymal sperm from WT and nGPx4-KO mice were capacitated and then allowed to in vitro fertilize metaphase ll oocytes that had previously been deprived of their cumulus cells and zona-pellucida and then pre-loaded with Hoechst 33342 
(a)
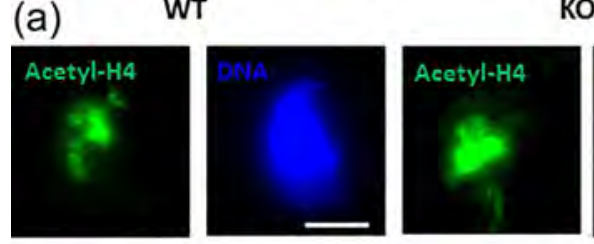

$\mathrm{KO}$

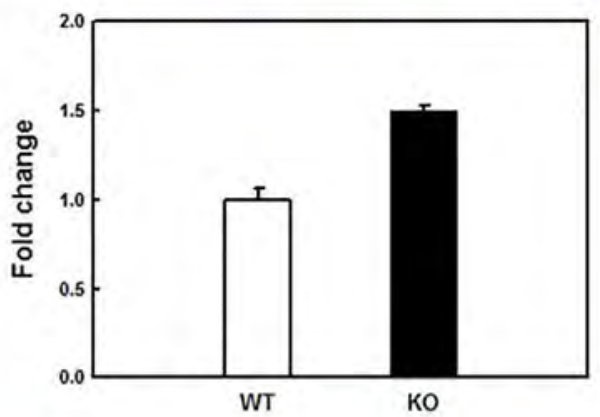

(b)

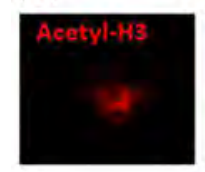
WT
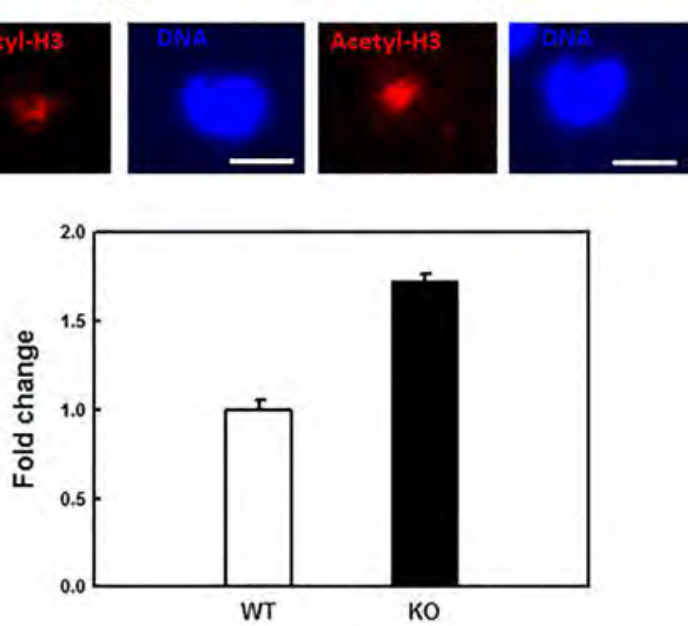

FIGURE 2 Immunofluorescence analysis of acetylated histone $\mathrm{H} 4$ (a) and acetylated histone $\mathrm{H} 3$ (b) of cauda epididymal sperm from WT and nGPx4-KO mice. Cells were subjected to the nuclear decondensation assay and then stained with anti-acetyl- $\mathrm{H} 4$ or antiacetyl-H3 antibodies. TO-PRO-3 was used to stain DNA. Histone signals were quantified by Image J software on sperm heads of the "medium" decondensation class. Note that nGPx4-KO sperm carry significantly higher levels of both acetylated histones compared to WT. Bars represent mean \pm SEM. Difference between WT and KO: acetyl-H4, $p<0.001$; acetyl-H3, $p<0.001$, calculated by Student's $t-$ test. Scale bars: $10 \mu \mathrm{m}$

(allowing to selectively stain the oocyte entered spermatozoa) in the presence or absence of TSA. The morphology of oocyte entered sperm was then analyzed $1 \mathrm{~h}$ after insemination according to the four levels of nuclear decondensation described above (Figure 4). In the absence of TSA treatment, the nuclei of $\mathrm{nGPx4-KO}$ sperm decondensed significantly faster than those of WT, in line with our previous observations on sperm chromatin decondensation in vitro (Puglisi et al., 2012). As for WT sperm, the presence of TSA caused a significantly higher frequency of the "high" decondensation class, indicating that an increased level of sperm histone acetylation caused a faster chromatin decondensation following penetration into oocyte. In contrast, sperm from nGPX4-KO mice did not show any decondensation change under TSA treatment, most likely because the extent of histone acetylation was already maximal. We therefore concluded that the process of paternal chromatin remodeling at fertilization is affected by the level of acetylated histones the sperm are equipped with.

\section{4 | The paternally imprinted gene Igf2/H19 ICR is hypomethylated in nGPX4-KO sperm}

In light of the abnormal histone acetylation pattern of nGPx4-KO sperm, we addressed the question of whether the epigenome of these sperm was also abnormal at the level of specific paternally imprinted genes. To this end, we analyzed the DNA methylation pattern of the imprinting control regions (ICR) of the paternally imprinted genes Igf $2 / \mathrm{H} 19$. We focused on two regions bearing CTCF binding sites, determining if CpG were differentially methylated in nGPx4-KO cauda epididymal sperm compared to WT. The methylation pattern of $42 \mathrm{CpG}$ sites is shown in Figure 5. nGPx4-KO sperm had the cytosine moieties in seven adjacent sites in fragment $A(444,447,461,468,481,579,632)$ and 3 adjacent sites in fragment $B(1915,1921,1923)$ significantly less methylated than those of WT sperm, indicating that errors in sperm epigenetic marks occur when chromatin assembly is perturbed.

\section{5 | nGPX4-KO mice display a decreased fertility caused by a significant delay in sperm ability to penetrate the zona pellucida}

The hypomethylation in the Igf2/H19 ICR locus of nGPx4-KO sperm was consistent with other defects observed, like sperm chromatin instability and abnormally high levels of histone acetylation. We therefore decided to gain more insights into the abnormal phenotype of these spermatozoa by determining the overall fertility of male nGPx4-KO mice. The mating ability was measured by crossing WT and nGPx4-KO males with hormone-primed CD1 females and then determining the percentage of plug-positive females resulting in pregnancies and the number of pups/litter (Table 1). The plug frequencies of the two genotypes were similar, indicating that nGPx4-KO mice had normal mating ability. These males, however, produced significantly less pregnancies to term and smaller litter sizes than WT males, indicating subfertility.

To further identify the defect(s) underlying the reduced fertilizing ability of nGPx4-KO males, we compared the early development of embryos resulting from the cross of both WT and nGPx4-KO males with hormone-primed CD1 females. Fertilized eggs were collected from the tubes $13 \mathrm{~h}$ after hCG injection and were then cultured in vitro for $12 \mathrm{~h}$, visually scoring the formation of pronuclei (PN) every $30 \mathrm{~min}$. Logistic curves of cumulative $\mathrm{PN}$ frequency obtained in this experiment are reported in Figure 6A. PN embryos from WT males first appeared at approximately $16.0 \mathrm{~h}$ post-hCG and maximized at approximately $22.0 \mathrm{~h}$ post-hCG. In contrast, PN embryos from nGPx4$\mathrm{KO}$ males first appeared at $17.0-17.5 \mathrm{~h}$ post-hCG and maximized at 

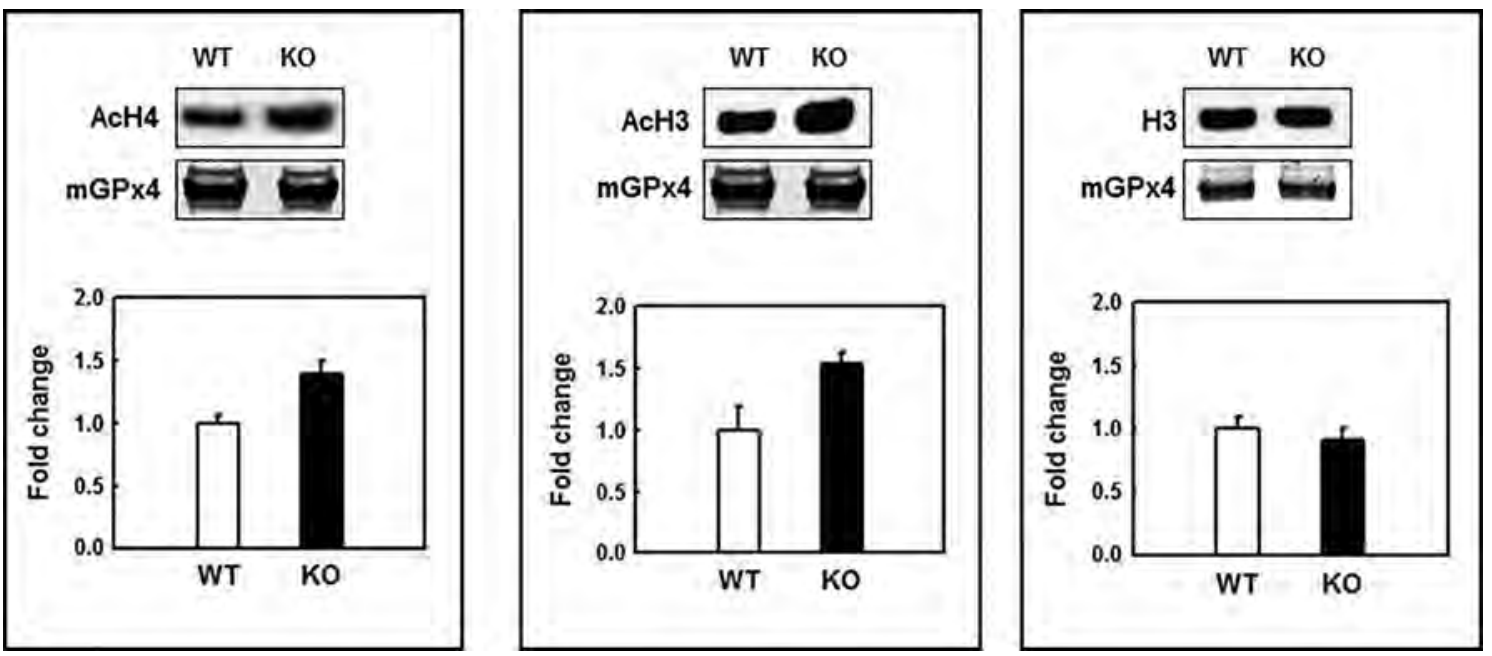

FIGURE 3 Western blot analysis of acetylated histone $\mathrm{H} 4$, acetylated histone $\mathrm{H} 3$, and unmodified histone $\mathrm{H} 3$ in cauda epididymal sperm from WT and nGPX4-KO mice. Sperm proteins were extracted, fractionated by AU-PAGE and analyzed by immunoblotting as described under materials and methods. Band densitometry was normalized to mitochondrial Gpx4 (mGPx4), revealing a significant increase of acetylated histones $\mathrm{H} 3$ and $\mathrm{H} 4$ in nGPx4-KO sperm compared to WT ones, $(p<0.05)$. In contrast, the two genotypes did not differ in their unmodified histone $\mathrm{H} 3$ content $(p>0.05)$. Bars represent mean \pm SEM. Differences were calculated by Student's $t$-test

approximately $23.0 \mathrm{~h}$ post-hCG. In addition to this delay, the two logistic curves also differed in terms of their maximal PN frequency (approximately $57 \%$ and $28 \%$ for WT and nGPx4-KO males, respectively), indicating that $\mathrm{nGPx} 4-\mathrm{KO}$ males produced significantly less embryos than WT ones. To get a more evident comparison

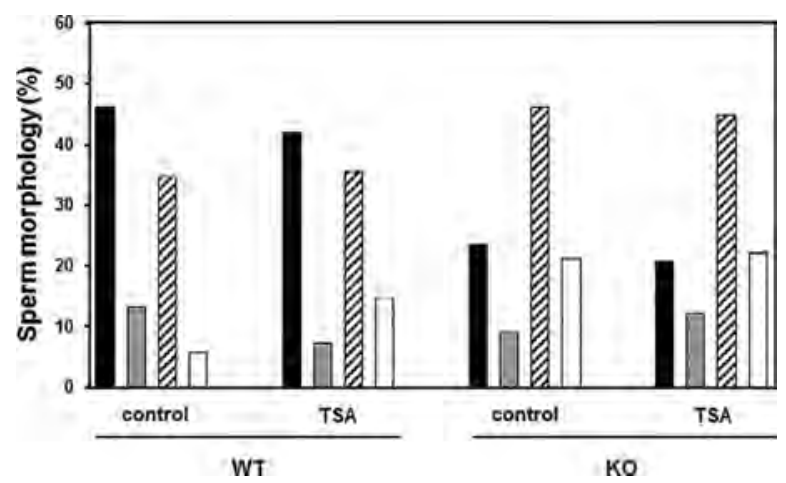

FIGURE 4 The presence of TSA in the medium causes a faster nuclear decondensation of WT, but not nGPx4-KO, sperm at fertilization. ZP-free metaphase II oocytes were pre-loaded with Hoechst and then incubated with capacitated cauda epididymal sperm from WT and nGPx4-KO mice, in the absence (control) or presence of TSA (TSA) to increase histone acetylation, as described under materials and methods. Based on their morphology, oocyteentered sperm were classified into the four classes of nuclear decondensation mentioned above: no decondensation, full bars; low decondensation, grey bars; medium decondensation, dashed bars; high decondensation, open bars. Sperm numbers for each class of morphology are expressed as percentage of total cells examined in five independent experiments. Data were statistically evaluated by chi square test: WT control vs WT TSA, $p<0.005$; nGPx4-KO control vs nGPx4-KO TSA, $p>0.5$; WT control vs nGPx4-KO control, $p<0.001$ between genotypes in terms of PN formation kinetics, the two logistic curves were normalized by expressing the mean values of panel $A$ as percentage of their maximal values (Figure 6B). The embryos from $\mathrm{nGPx} 4-\mathrm{KO}$ males reached the PN stage with a $2 \mathrm{~h}$ delay compared to those from WT ones, indicating that the lack of nGPx4 negatively affected the sperm ability to fertilize the egg. In addition, curves had similar rate of growth, suggesting similar kinetics of embryo development from fertilization to $\mathrm{PN}$ formation (Supplementary Figure S1).

To elucidate the cause(s) underlying the fertilization delay of nGPx4-KO sperm compared to WT, we devised experiments aimed at identifying the specific level(s) at which nGPx4 was actually required during fertilization. Putative fertilization steps to be investigated are: (i) sperm progression through cumulus and/or ZP, (ii) sperm binding to $Z P$, and (iii) sperm fusion with oolemma. We first ruled out the possibility that the $2 \mathrm{~h}$ delay merely represented a defective nGPx4$\mathrm{KO}$ sperm progression through female genital tracts, because nGPx4$\mathrm{KO}$ and WT sperm were similarly abundant in tubal fluid and oocyte cumuli at the time of their recovery from the tubes (data not shown). Next, we in vitro fertilized cumulus-enclosed CD1 eggs with capacitated sperm from nGPx4-KO or WT mice for $4 \mathrm{~h}$ and then determined the frequency of PN embryos after a further $4 \mathrm{~h}$ incubation. The PN embryos derived from nGPx4-KO sperm were dramatically less than those from WT sperm (Figure 7A), pinpointing the sperm-egg interaction per se as the point of interest for further investigation. We therefore investigated the nGPx4-KO and WT sperm ability to bind to ZP. This assay consisted in the in vitro incubation of capacitated nGPX4-KO and WT sperm with cumulusdeprived eggs for $1 \mathrm{~h}$. ZP-unbound spermatozoa were then gently washed out and the number of ZP-bound spermatozoa per oocyte was finally scored (Figure 7B). No difference in ZP-bound spermatozoa was observed between the two genotypes, ruling out the possibility that 
(1)

(a)

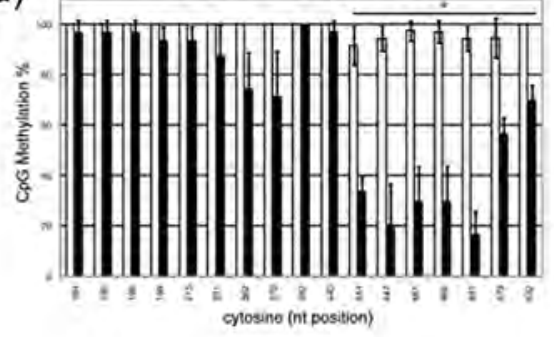

(b)

Ig/2/H19 DMR - fragment B

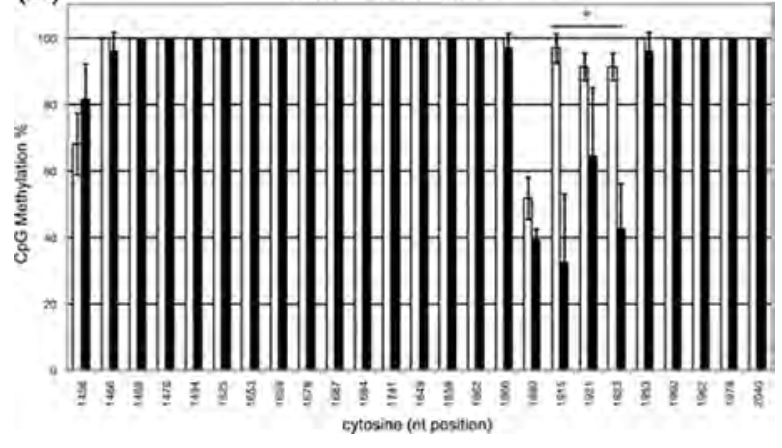

FIGURE 5 CpG methylation pattern of Igf2/H19 DMR in WT and nGPx4-KO sperm. CpG methylation pattern is expressed as methylation percentage for each $\mathrm{CpG}$ in two different regions (a and b) of Igf2/H19 DMR. Cytosine position in the reference sequence is reported under the bars, indicating the values (mean + SEM) obtained for WT (empty bars) and nGPx4-KO (full bars) sperm. WT vs $\mathrm{nGPX} 4-\mathrm{KO}$ in the regions indicated by asterisk, $p<0.02$. Significant difference was calculated by Mann-Wittney non-parametric test because the experimental method (sequencing of at least 10 clones for each experimental replicate) resulted in percent values (methylation \%) for many cytosines (non-correlated values) in each sample

sperm binding to ZP was a limiting factor. Finally, we compared spermoolemma fusion in both sperm genotypes by in vitro matching nGPx4KO and WT sperm with ZP-deprived and Hoechst 33342-loaded eggs. The number of Hoechst-positive sperm was then determined after 30 min incubation (Figure 7C). No difference was observed between the two genotypes, pinpointing the penetration through ZP as the fertilization step that was defective in $\mathrm{nGPx4-KO}$ sperm.

\section{4 | DISCUSSION}

In this study we provide new insights into the molecular underpinning of male pronucleus formation, proposing a mechanistic link between sperm retained acetylated histones and the decondensation of paternal chromatin inside the fertilized egg. The first step in the process of sperm nucleus decondensation is the disulfide bond reduction of protamines triggering their removal, and, consequently, their progressive replacement with histones, except at paternal chromatin regions already assembled in nucleosomes. Lastly the pronuclear DNA replication takes place. The general view regarding sperm nucleus decondensation is that the oocyte actively participates in the various steps of this process providing unlocking factors that reduce disulfide bonds, such as the thioredoxin Deadhead recently described in Drosophila (Tirmarche, Kimura, Dubruille, Horard, \& Loppin, 2016) and glutathione, similarly acting in mammals (Perreault, Barbee, \& Slott, 1988; Sutovsky \& Schatten, 1997). In addition the oocyte provides the machinery required for histone modification and incorporation onto the paternal chromatin. While the maternal origin of acetylated and methylated histones of the murine male pronucleus was supported by several studies (Santos et al., 2005; Torres-Padilla, Bannister, Hurd, Kouzarides, \& Zernicka-Goetz, 2006; van der Heijden et al., 2005, 2006), the paternal contribution to this process has remained unproven for a long time (Adenot et al., 1997; McLay \& Clarke, 2003; Spinaci, Seren, \& Mattioli, 2004), likely because the amount of histones associated to sperm chromatin is small and hardly accessible, particularly in the mouse (Gaucher et al., 2010; Hazzouri et al., 2000; Song et al., 2011; Wu, Caron, De, Khochbin, \& Rousseaux, 2008). In this study we have succeeded in revealing acetylated $\mathrm{H} 4$ and acetylated $\mathrm{H} 3$ histones in mature sperm isolated from adult mouse cauda epididymis by both immunofluorescence and western blot analyses, thus adding further evidence to a recent mass spectrometry analysis (Brunner, Nanni \& Mansuy, 2014). Consistent with this, the presence of acetylated histones was also demonstrated in both human and marmoset ejaculated sperm (Hecht et al., 2009; Krejci et al., 2015; Paradowska et al., 2012; Steilmann et al., 2011; van der Heijden et al., 2008).

Given that transcriptional and DNA replication activities of the male pronucleus initiate after this pronucleus is decondensed (Adenot et al., 1997; Bouniol, Nguyen, \& Debey, 1995; Paradowska et al., 2012), a specific role of paternal acetylated histones at the onset of

TABLE 1 Fertility of wild-type and nGPx4 KO male mice ${ }^{a}$

\begin{tabular}{|c|c|c|c|c|c|c|c|c|c|c|c|c|c|c|}
\hline \multirow[b]{2}{*}{ Animal number } & \multicolumn{7}{|c|}{ Wild-type } & \multicolumn{7}{|c|}{ nGPx4-KO } \\
\hline & 1 & 2 & 3 & 4 & 5 & 6 & mean \pm SEM & 7 & 8 & 9 & 10 & 11 & 12 & mean \pm SEM \\
\hline Plug number & 6 & 6 & 6 & 6 & 6 & 6 & $6.0 \pm 0.0^{b}$ & 6 & 6 & 6 & 6 & 5 & 6 & $5.8 \pm 0.1^{b}$ \\
\hline Pregnancy with delivery & 5 & 5 & 5 & 5 & 6 & 6 & $5.3 \pm 0.2^{c}$ & 4 & 2 & 5 & 5 & 2 & 3 & $3.5 \pm 0.6^{c}$ \\
\hline Total pup number & 53 & 58 & 55 & 55 & 72 & 85 & $63.0 \pm 5.2^{d}$ & 35 & 13 & 38 & 44 & 18 & 19 & $27.8 \pm 5.2^{d}$ \\
\hline
\end{tabular}

${ }^{a}$ The fertility of six wild-type and six nGPx4-KO males was assessed by sequentially mating each mouse with six CD1 hormone-primed females, as described under Materials and Methods; differences between means were calculated by Student's $t$-test.

$\mathrm{b}_{p}>0.3$.

${ }^{c} p<0.05$.

$\mathrm{d}_{p}<0.001$. 

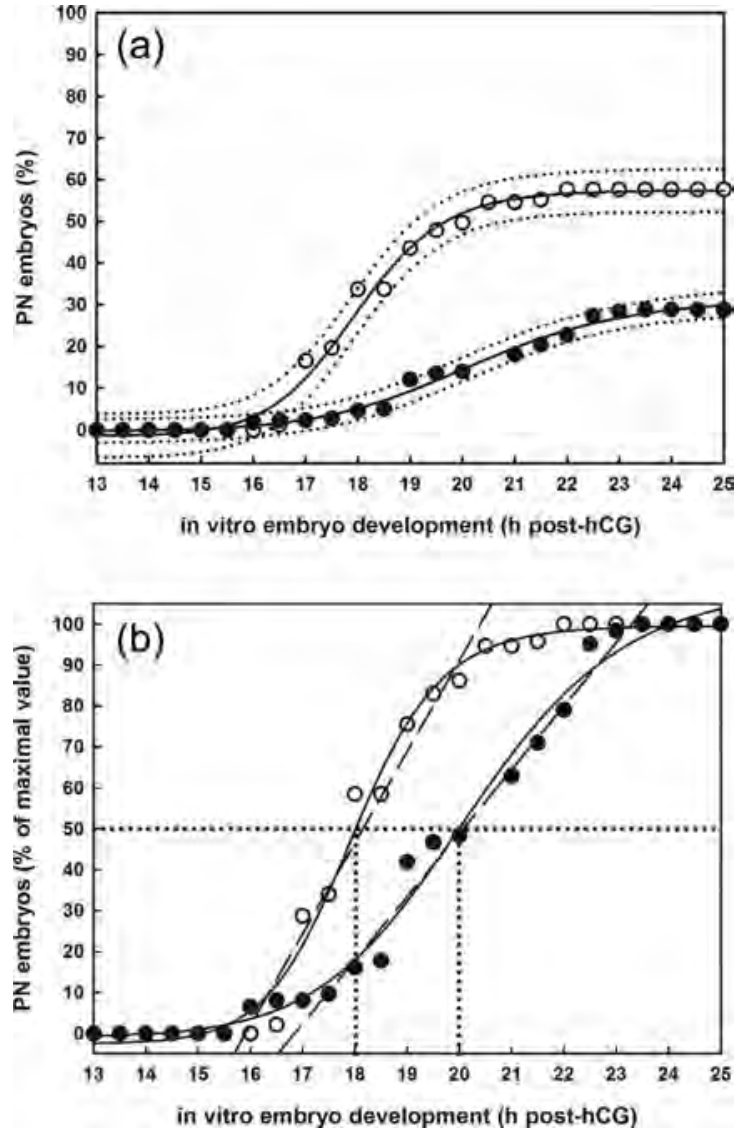

FIGURE 6 Analysis of the in vivo fertilizing ability of sperm from WT and nGPx4-KO mice. WT and nGPx4-KO males were mated with hormone-primed CD1 females and embryos produced were in vitro cultured for $12 \mathrm{~h}$, monitoring the formation of pronuclei (PN) every $30 \mathrm{~min}$, as described in the text. (a) Data pooled from 6 independent experiments were expressed as logistic curves of cumulative PN frequencies (calculated by Sigma Plot with the "logistic four parameters curve" function; dotted lines outline the $95 \%$ prediction bands). Note the difference in the timing of PN appearance and the approximately $50 \%$ reduction of the total embryos produced by nGPx4-KO sperm (full dots) compared to WT ones (open dots). (b) Data of panel (a) were normalized as percentage of their maximal value. Besides the $2 \mathrm{~h}$ difference (vertical dotted lines), the two curves had similar rates of growth, indicating similar kinetics of PN formation. Regression lines (dashed lines) were calculated in the following arbitrary intervals: WT, $h$ 16.0-21.0; nGPX4-KO, h 17.5-22.5. Difference between slopes: $p>0.1$, see supplementary Figure S1 for further details

decondensation remains elusive. The present finding that sperm having a higher proneness to decondense contain higher amounts of acetylated histones compared to WT gametes is intriguing and points to the idea that modified histones of paternal origin directly participate in the very early dynamics of male nuclear decondensation leading to male pronucleus formation. Moreover, the possibility that maternally provided histones are required for this initial step, as proposed in the past (Adenot et al., 1997; McLay, Carroll, \& Clarke, 2002), is now lessened by our observations that spermatozoa from nGPx4-KO mice decondense at a faster rate respect to WT in both the in vitro decondensation assay (Puglisi et al., 2012) and following sperm entry in the oocyte (present results), due to the chromatin instability caused by the lack of nGPx4. To further support the concept that histone acetylation is relevant to male chromatin remodeling after sperm penetration in the oocyte we have performed in vitro fertilization experiments in which the level of sperm histone acetylation was increased by oocyte treatment with TSA. Our results have shown that TSA-treated WT sperm decondense faster than the untreated ones, whereas the decondensation of nGPx4-KO sperm was unaffected by TSA treatment, indicating a direct relationship between the amount of acetylated histones and male chromatin decondensation rate at fertilization. Indeed, it is of interest that also in a different mouse model displaying perturbed poly-(ADP-ribose) (PAR) metabolism, sperm are characterized by reduced nuclear condensation associated with elevated retention of modified histones (Meyer-Ficca et al., 2011; Puglisi et al., 2012). Present data are also supported by the fact that, while both oocytes and spermatozoa contain histone acetyltransferases, the enzymes responsible for histone acetylation (Lahn et al., 2002; McGraw, Morin, Vigneault, Leclerc, \& Sirard, 2007), mature mouse sperm, but not oocytes, lack histone deacetylases (Hazzouri et al., 2000). It thus appears that acetylation/deacetylation activities are unbalanced in the spermatozoon, guaranteeing the male chromatin to be provided of an adequate equipment of acetylated histones made during spermiogenesis. Reinforcing this idea, a TSA treatment of isolated epididymal sperm had no effect on the rate of chromatin decondensation in our in vitro decondensation assay (data not shown). Taken together, these data strongly argue in favor of a critical structural role of sperm acetylated histones in modulating the transition from the highly compact state of paternal chromatin to the decondensed one following fertilization.

Another goal of our study was to gain more insights into the defective phenotype of $\mathrm{nGPx} 4-\mathrm{KO}$ sperm and its functional consequences. Interestingly, our finding that ICRs of Igf $2 / \mathrm{H} 19$ paternally imprinted genes were significantly hypomethylated in the mutant sperm compared to WT unveils an additional epigenetic error in these cells, besides the altered acetylation of retained histones. Histone acetylation and DNA hypomethylation are likely two players of the same mechanism, acting in combination and leading to male chromatin decondensation. As we previously demonstrated that $\mathrm{nGPx4-KO}$ sperm chromatin is unstable and more prone to decondense (Puglisi et al., 2012), collectively our data provide further sound evidence for the notion that sperm abnormal chromatin packaging is associated with alteration of chromatin composition and epigenetic profile. In line with this, DNA methylation defects were observed in sperm from men with abnormal protamine1/protamine 2 ratio (Aston, Punj, Liu, \& Carrell, 2012) and aberrant DNA methylation of Igf2/H19 locus was also reported in spermatozoa from oligo- and teratozoospermic men (Boissonnas et al., 2010; Poplinski, Tuttelmann, Kanber, Horsthemke, \& Gromoll, 2010).

Within this framework, we have also observed a relationship between disruption of sperm epigenetic feature and fertility. Indeed, as one would predict, male $\mathrm{nGPx4-KO}$ mice were found to be subfertile. We have carefully assessed the fertility of the mutant mice in both in vivo and in vitro experiments, revealing a previously unrecognized role 

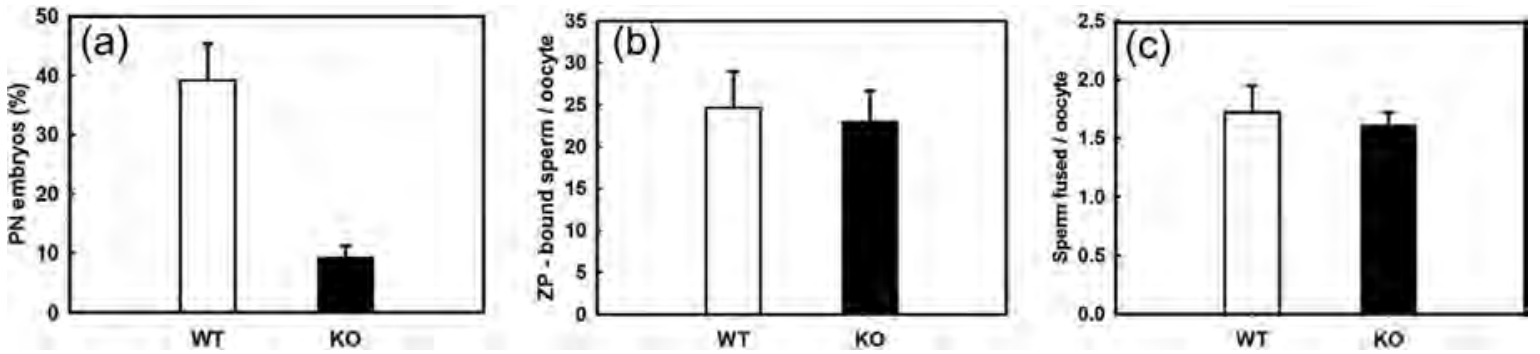

FIGURE 7 Analysis of the in vitro fertilizing ability of sperm from WT and nGPx4-KO mice. (a) In vitro fertilization: cumulus-enclosed oocytes were incubated in vitro with WT or nGPx4-KO sperm for $4 \mathrm{~h}$, then washed, in vitro cultured for additional $4 \mathrm{~h}$ and finally scored for PN formation. Note the reduction of PN embryos observed with nGPx4-KO vs WT sperm $(p<0.001)$. (b) Zona pellucida binding assay: WT and nGPX4-KO sperm ability to bind the zona pellucida was evaluated as described under Materials and Methods. No difference was observed between the sperm of the two genotypes $(p>0.7)$. (c) In vitro fertilization assay with zona pellucida-free oocytes: CD1 oocytes were deprived of their ZP, loaded with Hoechst 33342 and in vitro fertilized with WT or nGPx4-KO sperm, as described under Materials and Methods. WT and nGPx4-KO sperm displayed similar ability to fuse with oolemma $(p>0.6)$. Bars of panels $(a-c)$ indicate the mean + SEM of values obtained in at least three independent experiments. Differences between WT and KO was calculated by Student's $t$-test

for nGPx4 in male fertility (Conrad et al., 2005). We addressed whether sperm lacking nGPx4 had fertility defect(s) under physiological conditions, allowing WT and mutant sperm to fertilize eggs in vivo and then determining the frequency and early developmental kinetics of resulting embryos in vitro. The frequency accumulation curves obtained strikingly differed with each other, indicating that the lack of sperm nGPx4 caused an approximately $50 \%$ reduction in the number of PN embryos compared to WT. That this reduction directly depended on fertilization rate per se while the early embryo development was normal, became apparent when the two frequency curves were normalized with each other, giving similar slopes and thus similar post-fertilization kinetics, but significantly different onsets, indicating that $\mathrm{nGPx} 4-\mathrm{KO}$ sperm entered the eggs $2 \mathrm{~h}$ later than WT sperm did. An incoming zona pellucida hardening, caused by spontaneous cortical granule release (Hoodbhoy \& Talbot, 1994) during egg overriping at such late fertilization times, is likely involved in the striking reduction in the number of $\mathrm{PN}$ embryos derived from mutant sperm. We have further investigated the fertilization delay of $n G P x 4-K O$ sperm by in vitro experiments, showing that nGPx4-KO sperm performed normally in two crucial steps of fertilization process, namely the binding to zona pellucida and the fusion with egg membrane. It thus appears that mutant sperm have a specific failure in their progression through cumulus/ zona pellucida, a process which requires a functional acrosome and acrosomal reaction (Yanagimachi, 2011). In this context, it is interesting to note that the nuclear isoform of glutathione peroxidase has two different localizations in sperm head: the acrosome and the nuclear matrix (Puglisi et al., 2012). We previously demonstrated that acrosomal nGPx4 is stably associated with the acrosomal matrix, an insoluble sperm structure involved in the zona pellucida penetration during fertilization (Muro, Buffone, Okabe, \& Gerton, 2012; Foster \& Gerton, 2016). We therefore hypothesize that acrosomal nGPx4 is needed for the normal occurrence of acrosomal reaction. Whether the defect caused by the lack of such nGPx4 takes place at the specific level of cumulus and/or zona pellucida and its molecular basis remains to be investigated by further studies. Given the well documented importance of selenium for mammalian male fertility and keeping in mind that the entire sperm selenium content is associated with Glutathione Peroxidase 4, our data provide new insights into the roles the isoforms and subcellular localization of this enzyme carry out, namely: (i) the mitochondrial form, mGPx4, is needed for sperm motility (Schneider et al., 2009); (ii) the nuclear form associated with nuclear matrix plays a role in sperm chromatin compaction during spermiogenesis and the regulation of the level of acetylated histones in mature sperm; and (iii) the nuclear form associated with acrosomal matrix is required for acrosome structure and acrosomal reaction at fertilization.

In summary, our data shed light on mechanisms for the early remodeling of paternal chromatin at fertilization, providing new evidence of the contribution of sperm retained acetylated histones, and reveal a novel role played by the nuclear isoform of GPx4 in male fertility.

\section{ACKNOWLEDGMENTS}

We are grateful to Dr. Marcus Conrad for providing nGPx4-KO mice. We thank Dr. Paola Caporali for help with statistical analysis and Ms Tiziana Menna for her technical assistance. This work was supported by grant from the Italian Ministry of Education.

\section{CONFLICTS OF INTEREST}

The authors have nothing to disclose and declare that there is no conflict of interest.

\section{ORCID}

Carla Boitani (10) http://orcid.org/0000-0002-8452-8907

\section{REFERENCES}

Adenot, P. G., Mercier, Y., Renard, J. P., \& Thompson, E. M. (1997). Differential $\mathrm{H} 4$ acetylation of paternal and maternal chromatin precedes DNA replication and differential transcriptional activity in pronuclei of 1-cell mouse embryos. Development, 124, 4615-4625. 
Arpanahi, A., Brinkworth, M., lles, D., Krawetz, S. A., Paradowska, A., Platts, A. E., ... Miller, D. (2009). Endonuclease-sensitive regions of human spermatozoal chromatin are highly enriched in promoter and CTCF binding sequences. Genome Research, 19, 1338-1349.

Aston, K. I., Punj, V., Liu, L., \& Carrell, D. T. (2012). Genome-wide sperm deoxyribonucleic acid methylation is altered in some men with abnormal chromatin packaging or poor in vitro fertilization embryogenesis. Fertility and Sterility, 97, 285-292.

Boissonnas, C. C., Abdalaoui, H. E., Haelewyn, V., Fauque, P., Dupont, J. M. Gut, I., ... Jammes, H. (2010). Specific epigenetic alterations of IGF2$\mathrm{H} 19$ locus in spermatozoa from infertile men. European Journal of Human Genetics, 18, 73-80.

Bonnefoy, E., Orsi, G. A., Couble, P., \& Loppin, B. (2007). The essential role of Drosophila HIRA for de novo assembly of paternal chromatin at fertilization. PLoS Genetics, 3, 1991-2006.

Bouniol, C., Nguyen, E., \& Debey, P. (1995). Endogenous transcription occurs at the 1-cell stage in the mouse embryo. Experimental Cell Research, 218, 57-62.

Brunner, A. M., Nanni, P., \& Mansuy, I. M. (2014). Epigenetic marking of sperm by post-translational modification of histones and protamines. Epigenetics Chromatin, 7, 2.

Brykczynska, U., Hisano, M., Erkek, S., Ramos, L., Oakeley, E. J., Roloff, T. C., .. Peters, A. H. (2010). Repressive and active histone methylation mark distinct promoters in human and mouse spermatozoa. Nature Structural and Molecular Biology, 17, 679-687.

Chen, Q., Yan, W., \& Duan, E. (2016). Epigenetic inheritance of acquired traits through sperm RNAs and sperm RNA modifications. Nature Reviews Genetics, 17, 733-743.

Conrad, M., Moreno, S. G., Sinowatz, F., Ursini, F., Kolle, S., Roveri, A., . . Bornkamm, G. W. (2005). The nuclear form of phospholipid hydroperoxide glutathione peroxidase is a protein thiol peroxidase contributing to sperm chromatin stability. Molecular and Cellular Biology, 25, 7637-7644.

Fiorenza, M. T., Torcia, S., Canterini, S., Bevilacqua, A., Narducci, M. G., Ragone, G., ... Mangia, F. (2008). TCL1 promotes blastomere proliferation through nuclear transfer, but not direct phosphorylation, of AKT/PKB in early mouse embryos. Cell Death and Differentiation, 15 , 420-422.

Foster, J. A., \& Gerton, G. L. (2016). The acrosomal matrix. Advances in Anatomy, Embryology and Cell Biology, 220, 15-33.

Fuso, A., Ferraguti, G., Scarpa, S., Ferrer, I., \& Lucarelli, M. (2015). Disclosing bias in bisulfite assay: MethPrimers underestimate high DNA methylation. PLoS ONE, 10, e0118318.

Fuso, A., Nicolia, V., Pasqualato, A., Fiorenza, M. T., Cavallaro, R. A., \& Scarpa, S. (2011). Changes in Presenilin 1 gene methylation pattern in diet-induced B vitamin deficiency. Neurobiology of Aging, 32, 187-199.

Fuso, A., Scarpa, S., Grandoni, F., Strom, R., \& Lucarelli, M. (2006). A reassessment of semiquantitative analytical procedures for DNA methylation: Comparison of bisulfite- and Hpall polymerase-chainreaction-based methods. Analytical Biochemistry, 350, 24-31.

Gaucher, J., Reynoird, N., Montellier, E., Boussouar, F., Rousseaux, S., \& Khochbin, S. (2010). From meiosis to postmeiotic events: The secrets of histone disappearance. FEBS Journal, 277, 599-604

Hammoud, S. S., Nix, D. A., Zhang, H., Purwar, J., Carrell, D. T., \& Cairns, B. R. (2009). Distinctive chromatin in human sperm packages genes for embryo development. Nature, 460, 473-478.

Hammoud, S. S., Purwar, J., Pflueger, C., Cairns, B. R., \& Carrell, D. T. (2010) Alterations in sperm DNA methylation patterns at imprinted loci in two classes of infertility. Fertility and Sterility, 94, 1728-1733.

Hazzouri, M., Pivot-Pajot, C., Faure, A. K., Usson, Y., Pelletier, R., Sele, B., . . . Rousseaux, S. (2000). Regulated hyperacetylation of core histones during mouse spermatogenesis: Involvement of histone deacetylases. European Journal of Cell Biology, 79, 950-960.

Hecht, N., Behr, R., Hild, A., Bergmann, M., Weidner, W., \& Steger, K. (2009). The common marmoset (Callithrix jacchus) as a model for histone and protamine expression during human spermatogenesis. Human Reproduction, 24, 536-545.

Hoodbhoy, T., \& Talbot, P. (1994). Mammalian cortical granules: Contents, fate, and function. Molecular Reproduction and Development, 39 , 439-448.

Jenkins, T. G., \& Carrell, D. T. (2012). The sperm epigenome and potential implications for the developing embryo. Reproduction, 143, 727-734.

Krejci, J., Stixova, L., Pagacova, E., Legartova, S., Kozubek, S., Lochmanova, G., . . Bartova, E. (2015). Post-translational modifications of histones in human sperm. Journal of Cellular Biochemistry, 116, 2195-2209.

Lahn, B. T., Tang, Z. L., Zhou, J., Barndt, R. J., Parvinen, M., Allis, C. D., .. . Page, D. C. (2002). Previously uncharacterized histone acetyltransferases implicated in mammalian spermatogenesis. Proceedings of the National Academy of Sciences of the United States of America, 99, 8707-8712.

Lin, Y. N., Roy, A., Yan, W., Burns, K. H., \& Matzuk, M. M. (2007). Loss of zona pellucida binding proteins in the acrosomal matrix disrupts acrosome biogenesis and sperm morphogenesis. Molecular and Cellular Biology, 27, 6794-6805.

Luconi, M., Torcia, S., Grillo, D., Fiorenza, M. T., Forti, G., Mangia, F., \& Baldi, E. (2005). Enhancement of mouse sperm motility by the PI3-kinase inhibitor LY294002 does not result in toxic effects on preimplantation embryo development. Human Reproduction, 20, 3500-3504.

Luense, L. J., Wang, X., Schon, S. B., Weller, A. H., Lin, S. E., Bryant, J. M., . . Berger, S. L. (2016). Comprehensive analysis of histone posttranslational modifications in mouse and human male germ cells. Epigenetics Chromatin, 9, 24.

McGraw, S., Morin, G., Vigneault, C., Leclerc, P., \& Sirard, M. A. (2007). Investigation of MYST4 histone acetyltransferase and its involvement in mammalian gametogenesis. BMC Developmental Biology, 7, 123.

McLay, D. W., Carroll, J., \& Clarke, H. J. (2002). The ability to develop an activity that transfers histones onto sperm chromatin is acquired with meiotic competence during oocyte growth. Developmental Biology, 241, 195-206.

McLay, D. W., \& Clarke, H. J. (2003). Remodelling the paternal chromatin at fertilization in mammals. Reproduction, 125, 625-633.

Meistrich, M. L. (1989). Histone and basic nuclear protein transitions in mammalian spermatogenesis. Histones and other basic nuclear proteins. Orlando, Flo: CRC Press, (165-182)

Meyer-Ficca, M. L., Ihara, M., Lonchar, J. D., Meistrich, M. L., Austin, C. A., Min, W., ... Meyer, R. G. (2011). Poly(ADP-ribose) metabolism is essential for proper nucleoprotein exchange during mouse spermiogenesis. Biology of Reproduction, 84, 218-228.

Muro, Y., Buffone, M. G., Okabe, M., \& Gerton, G. L. (2012). Function of the acrosomal matrix: Zona pellucida 3 receptor (ZP3R/sp56) is not essential for mouse fertilization. Biology of Reproduction, 86, 1-6.

Nagy, A., Gertsenstein, M., Vintersten, K., \& Behringer, R. (2003). Manipulating the mouse embryo. Cold Spring Harbor Laboratory Press.

Paradowska, A. S., Miller, D., Spiess, A. N., Vieweg, M., Cerna, M., Dvorakova-Hortova, K., .. . Steger, K. (2012). Genome wide identification of promoter binding sites for $\mathrm{H} 4 \mathrm{~K} 12 \mathrm{ac}$ in human sperm and its relevance for early embryonic development. Epigenetics, 7, 1057-1070.

Perreault, S. D., Barbee, R. R., \& Slott, V. L. (1988). Importance of glutathione in the acquisition and maintenance of sperm nuclear decondensing activity in maturing hamster oocytes. Developmental Biology, 125, 181-186.

Poplinski, A., Tuttelmann, F., Kanber, D., Horsthemke, B., \& Gromoll, J. (2010). Idiopathic male infertility is strongly associated with aberrant methylation of MEST and IGF2/H19 ICR1. International Journal of Andrology, 33, 642-649.

Puglisi, R., Maccari, I., Pipolo, S., Conrad, M., Mangia, F., \& Boitani, C. (2012). The nuclear form of glutathione peroxidase 4 is associated with sperm nuclear matrix and is required for proper paternal chromatin 
decondensation at fertilization. Journal of Cellular Physiology, 227 1420-1427.

Rajender, S., Avery, K., \& Agarwal, A. (2011). Epigenetics, spermatogenesis, and male infertility. Mutation Research, 727, 62-71.

Rodgers, A. B., Morgan, C. P., Leu, N. A., \& Bale, T. L. (2015). Transgenerational epigenetic programming via sperm microRNA recapitulates effects of paternal stress. Proceedings of the National Academy of Sciences of the United States of America, 112, 13699-13704.

Santos, F., Hendrich, B., Reik, W., \& Dean, W. (2002). Dynamic reprogramming of DNA methylation in the early mouse embryo. Developmental Biology, 241, 172-182.

Santos, F., Peters, A. H., Otte, A. P., Reik, W., \& Dean, W. (2005). Dynamic chromatin modifications characterise the first cell cycle in mouse embryos. Developmental Biology, 280, 225-236.

Schneider, M., Forster, H., Boersma, A., Seiler, A., Wehnes, H., Sinowatz, F., ... Conrad, M. (2009). Mitochondrial glutathione peroxidase 4 disruption causes male infertility. FASEB Journal, 23, 3233-3242.

Siklenka, K., Erkek, S., Godmann, M., Lambrot, R., McGraw, S., Lafleur, C., . . . Kimmins, S. (2015). Disruption of histone methylation in developing sperm impairs offspring health transgenerationally. Science, 350, aab2006.

Song, N., Liu, J., An, S., Nishino, T., Hishikawa, Y., \& Koji, T. (2011). Immunohistochemical analysis of histone $\mathrm{H} 3$ modifications in germ cells during mouse spermatogenesis. Acta Histochemica et Cytochemica, 44, 183-190.

Sonnack, V., Failing, K., Bergmann, M., \& Steger, K. (2002). Expression of hyperacetylated histone $\mathrm{H} 4$ during normal and impaired human spermatogenesis. Andrologia, 34, 384-390.

Spinaci, M., Seren, E., \& Mattioli, M. (2004). Maternal chromatin remodeling during maturation and after fertilization in mouse oocytes. Molecular Reproduction and Development, 69, 215-221.

Steilmann, C., Paradowska, A., Bartkuhn, M., Vieweg, M., Schuppe, H. C., Bergmann, M., ... Steger, K. (2011). Presence of histone H3 acetylated at lysine 9 in male germ cells and its distribution pattern in the genome of human spermatozoa. Reproduction, Fertility, and Development, 23, 997-1011.

Sutovsky, P., \& Schatten, G. (1997). Depletion of glutathione during bovine oocyte maturation reversibly blocks the decondensation of the male pronucleus and pronuclear apposition during fertilization. Biology of Reproduction, 56, 1503-1512.

Tirmarche, S., Kimura, S., Dubruille, R., Horard, B., \& Loppin, B. (2016). Unlocking sperm chromatin at fertilization requires a dedicated egg thioredoxin in Drosophila. Nature Communications, 7, 13539.
Torres-Padilla, M. E., Bannister, A. J., Hurd, P. J., Kouzarides, T., \& ZernickaGoetz, M. (2006). Dynamic distribution of the replacement histone variant $\mathrm{H} 3.3$ in the mouse oocyte and preimplantation embryos. International Journal of Developmental Biology, 50, 455-461.

van der Heijden, G. W., Derijck, A. A., Ramos, L., Giele, M., van der Vlag, J., \& de Boer, P. (2006). Transmission of modified nucleosomes from the mouse male germline to the zygote and subsequent remodeling of paternal chromatin. Developmental Biology, 298, 458-469.

van der Heijden, G. W., Dieker, J. W., Derijck, A. A., Muller, S., Berden, J. H., Braat, D. D., ... de Boer, P. (2005). Asymmetry in histone H3 variants and lysine methylation between paternal and maternal chromatin of the early mouse zygote. Mechanisms of Development, 122, 1008-1022.

van der Heijden, G. W., Ramos, L., Baart, E. B., van den Berg, I. M., Derijck, A. A., van der Vlag, J., ... de Boer, P. (2008). Sperm-derived Histones Contribute to Zygotic Chromatin in Humans. BMC Developmental Biology, 8, 34.

Ward, W. S. (2010). Function of sperm chromatin structural elements in fertilization and development. Molecular Human Reproduction, 16, 30-36.

Wu, F., Caron, C., De, R. C., Khochbin, S., \& Rousseaux, S. (2008). Testisspecific histone variants H2AL1/2 rapidly disappear from paternal heterochromatin after fertilization. Journal of Reproduction and Development, 54, 413-417.

Wykes, S. M., \& Krawetz, S. A. (2003). The structural organization of sperm chromatin. Journal of Biological Chemistry, 278, 29471-29477.

Yanagimachi, R. (2011). Mammalian sperm acrosome reaction: Where does it begin before fertilization? Biology of Reproduction, 85, 4-5.

\section{SUPPORTING INFORMATION}

Additional Supporting Information may be found online in the supporting information tab for this article.

How to cite this article: Pipolo S, Puglisi R, Mularoni V, et al. Involvement of sperm acetylated histones and the nuclear isoform of Glutathione peroxidase 4 in fertilization. J Cell Physiol. 2017;1-12. https://doi.org/10.1002/jcp.26146 